\title{
Focusing and Acceleration of Bunched Beams
}

\author{
Zohreh. Parsa \\ Brookhaven National Laboratory \\ Physics Department \\ Upton, New York \\ and \\ Vladimir Zadorozhny \\ Inst. of Cybernetic, National Academy of Sciences of Ukraine
}

April 2000 


\title{
Focusing and Acceleration of Bunched Beams
}

\author{
Z. Parsia ${ }^{1}$ \\ Brookhaven National Laboratory, Physics Department 510 A, Upton, NY 11973 \\ parsa@bnl.gov \\ V. Zadorozhny
}

Institute of Cybernetic, National Academy of Sciences of Ukraine

zvf@umex.istrada.net.ua

\begin{abstract}
A new approach to solving the kinetic equation for the beam distribution function, (very useful from the practical point of view), is discussed. In which we also obtain a complement to the Skrinsky's condition for the self-focused bunched beam. This problem belongs to the theory of nonlinear systems in which both regular and chaotic motion is possible. 'The kinetic approach, based on Vlasov-Poisson equations, are used to investigate the focusing and acceleration of bunched beam. Special attention is given to the studies of stability in a bunched beam by means of the two norm, which may be used to describe the motion of high - energy particles.
\end{abstract}

\section{INTRODUCTION}

The localized physical structure such as bunched beam, halo beam, vortex, etc.; can be represented in the form of soliton-like solution of some evolution equations, by hydrodynamic or kinetic approach. In accordance with contemporary studies the soliton-like solution arises due to a sufficiently large influence of external forces and also because of nonlinear effect of self-influence. Objects, which are formed in this case, have unusual properties. The selfconsistent Vlasov equation [1] is one of the most frequently used equations for the time dependent description of many- particle systems. By varying the selfconsistent Vlasov equation we can find different analytical solutions by means of which, its phase portraits can be studied.The selfconsistent Vlasov equation is a partial differential equation of a first order and the time evolution of its solutions are entirely determined by the initial distribution function. This property will be used below to organize the localized structure. It may be noted that the localized structures in a finite region

1) Supported by US Department of Energy contract DE-AC02-98CH10886 
are integrable models. The construction of such solutions will have physical sense if and only if they will be stable (in some sense), and obviously, every asymptotically stable solution is a self - focused solution. As well known the notation stable may vary as defined in various works (papers), for clarity in the case under consideration we will appeal to the notation of Lyapunov (stable) and its modifications only. That is, we consider 1) the stable solution of Vlasov equation having relation with an initial value of a position and velocity of some particles. 2) The stable solution of Vlasov equation as stable initial-value problem for the initial perturbs of an initial value for the beam distribution function. 3) The stable solution of Vlasov-Poisson equation with respect to action of the corresponding magnetic field. The exact sense of these notations will be established below.

Always there exist an electro-magnetic field satisfying the Maxwell equation for a given arbitrary motion, i.e. any motion of the beam may be realized by an electro-magnetic field which satisfy the Maxwell equation.

In contemporary physical World there are many interesting and challenging problems that may be resolved by solutions of Vlasov equation. For example, the motion of the muon bunches into a collider.

In a muon collider complex, a rapid acceleration to the collider beam energy is needed to avoid expensive particle loss from the muon decays. It can be achieved, initially in a linear accelerator and later in recirculating linear accelerators, rapidcycling synchrotron, or fixed - field - alternating - gradient accelerators. Positive and negative muon bunches are then injected in opposite directions into a collider storage ring and brought into collision at the interaction point. The bunches circulatc and collide for many revolutions before decay has depleted the beam intensities to an interesting level. Useful luminosity can be delivered for about 1000 revolutions for a high-energy (e.g. $\sim 4 \mathrm{TeV}$ ) collider and about 450 revolutions for a low-energy $(e . g . \sim 100 \mathrm{GeV})$ one [4].

Since Vlasov equation has high degree of confidence for sufficiently small time of Beam motion, given the limited muon life time $\left(\tau_{\mu}=2.2_{\mu s}\right.$ at rest), the Vlasov equation well describes the muon beam dynamics. In this approach, design concepts for acceleration and strong focusing of the muon beam are being developed. Now, we can perform study of the nonlinear motion of the muon bunches by VlasovMaxwell system.

\section{PRELIMINARIES}

We are going to consider the motion of many particles in the following standard form

$$
\dot{x}=v, \quad \dot{p}=q E+\frac{q}{c}(v \cdot H)
$$

here $p=m v$ is impulse of the particle, $v=\left(v_{1}, v_{2}, v_{3}\right)$ is the velocity, $x=$ $\left(x_{1}, x_{2}, x_{3}\right)$ is the vector which characterizes the position of this particlc in (Euclid) space $\left\{x_{1}, x_{2}, x_{3}\right\}$, i.e. $\left(x_{1}, x_{2}, x_{3}\right)$ is the coordinate system, $q$ is the charge of it, $m$ 
is the mass, $E$ is the electric field and $H$ denotes the magnetic field. The fields $E$ and $H$ are generated by a charged bunch and current, $E_{j}, H_{j}$ (here $j=1, \ldots k$ ) are quantity of the beams. If there are some outward fields $E_{0}$ and $H_{0}$ then

$$
E=\sum_{j=0}^{k} E_{j}, \quad H=\sum_{j=0}^{k} H_{j} .
$$

Let $\Omega_{t_{0}}$ be any set of the phase space $E_{0}=\{x, p\}$. If $\Omega_{t_{0}} \in E_{0}$ and $\left\{x_{t}\left(x_{0}\right), p_{t}\left(p_{0}\right)\right\}$ some solution of equation (1) for $\left(x_{0}, p_{0}\right) \in \Omega_{t_{0}}$ then obviously $\Omega_{t}=\left\{x_{t}\left(x_{0}\right), p_{t}\left(p_{0}\right) ;\left(x_{0}, p_{0}\right) \in \Omega_{t_{0}}\right\}$. Let $\rho(t, x, p)$ be some function such that it yields a simple equation

$$
\int_{\Omega_{t_{0}}} \rho\left(t_{0}, x_{0}, p_{0}\right) d x_{0} d p_{0}=\int_{\Omega_{t}} \rho\left(t, x_{t}, p_{t}\right) d x_{t} d p_{t}
$$

for any domain $\Omega_{t_{0}}$, which we will call density (of the integral invariant). As is well known, if system (1) have some integral invariant with the density $\rho$ then the function $\rho(t, x, v)$ satisfies the following equation

$$
\partial_{t} \rho+\partial_{x} \rho v+\partial_{v} \rho\left[q E+\frac{q}{c}(v \cdot H)\right]+\rho \cdot \operatorname{div}\left(v, q E+\frac{q}{c}(v \cdot H)\right)=0
$$

but is easy to see that, $\operatorname{div}\left(v, q E+\frac{q}{c}(v \cdot H)\right) \equiv 0$, consequently

$$
\partial_{t} \rho+v \partial_{x} \rho+\left[q E-\frac{q}{c}(v \cdot H)\right] \partial_{v} \rho=0 .
$$

Further we denote $\rho$ by $f$ (for tradition), thus the following equation

$$
\partial_{t} f+v \partial_{x} f+\left[q E-\frac{q}{c}(v \cdot H)\right] \partial_{v} f=0
$$

is Vlasov equation which we will study.

Let us now find the solution $f(t, x, v)$ of Vlasov equation in the form

$$
f=f_{0}(x, v) e^{-i \omega t}
$$

Substituting (4) in (3), we get

$$
v \partial_{x} f_{0}+\left[q E-\frac{q}{c}(v \cdot H)\right] \cdot \partial_{v} f_{0}=i \omega f_{0}
$$

and an operator form of the formula (5) yields

$$
L f_{0}=i \omega f_{0}
$$

for unknown $f_{0}$, where $L$ is a linear operator in a given Hilbert space $L^{2}\left(\bar{\Omega}_{t_{0}}\right)$ which is generated by an operator

$$
L_{0} \bullet=v \partial_{x} \bullet+\left[q E-\frac{q}{c}(v \cdot H)\right] \partial_{v} \bullet
$$

The domain $D\left(L_{0}\right)$ of the operator $L_{0}$ is dense in the space $L_{0}^{2}\left(\Omega_{t_{0}}\right)$. Thus the divergence of the phase space $\{x, v\}$ is identically equal to zero then dynamical system for the operator $L$ retain some measure. Consequently [16] there is the solution (strong or weak) and $f_{0} \in L^{2}\left(\Omega_{t_{0}}\right)$. 


\section{BUNCHED BEAM IN ELECTRIC FIELD}

The Maxwell equation reduce to the form $\operatorname{rot} E=0$ if magnetic field vanishes, and $E=-\nabla U$, where $\mathrm{U}$ is an electro-potential. An ultra-relativistic driving beam can be represented by the following equations

$$
\begin{gathered}
\dot{x}=v \\
(m \dot{v})=q E .
\end{gathered}
$$

Here $x=\left(x_{1}, x_{2}, x_{3}\right)$ is a position vector, $v=\left(v_{1}, v_{2}, v_{3}\right)$ is a velocity vector of $q$ is the beam particle charge, respectively. We suppose that a velocity field is given in the position space such that

$$
\dot{x}=\eta(x)
$$

Next we consider problem of existence of some field $E$ such that the motion of the beam will be identical to the motion given by (8), for case when initial values coincide. The statement becomes apparent when it is considered as the following. The equation (7) have an integral manifold

$$
v-\eta(x)==0
$$

Introducing into consideration the vector function

$$
\Theta=v-\eta(x)
$$

and calculating its time derivative with respect to the given equation (7) we get

$$
\dot{\Theta}=F(x, \Theta) .
$$

Obviously $F(x, \Theta) \equiv 0$ for $\Theta=0$. Let us consider (10) together with the equation (7). In accordance with existence and uniqueness Theorem for ordinary differential equations any solution with initial data $x=x_{0}, v=v_{0}, \Theta=\Theta_{0}$ for $t=0$ has the trivial solution $\Theta \equiv 0$ if $\Theta_{0}=0$. This means that equation (7) has integral manifold (9). In other words, if $v_{0}=\eta\left(x_{0}\right)$ as $t=0$ then $v=\eta(x)$ for all $t$.

Thus, the solution of the equation (7) satisfies also the equation (8) and consequently it is coinciding with its solution. Now let us consider the following problem: Suppose the integral manifold $\Theta$ and the distribution of velocity $\eta(v)$ on it is given. To find the field $E$ which is focusing and accelerate the beam for all $\left\{x_{0}, v_{0}\right\} \notin \Theta$, let us consider cquation (10) and corresponding to it the Cauchy problem for equation

$$
\frac{\partial V}{\partial \Theta} F=w, \quad V(0)=0
$$


Thus $\frac{d V}{d t} \equiv \frac{\partial V}{\partial \Theta} F$ with respect to the given system (10) and the function $w$ is to be such that the following holds

$$
\int_{0}^{\infty} w\left(\Theta_{t}\left(\Theta_{0}\right)\right) d t<\infty
$$

Then $V\left(\Theta_{t}\right)=V\left(\Theta_{0}\right)-\int_{0}^{\infty} w\left(\Theta_{t}\left(\Theta_{0}\right)\right) d t$. Here $Q_{0}=v-\eta(x)$ for $t=t_{0}$ and $Q_{t}\left(\Theta_{0}\right)$ is solution to equation (10). Although, we have neither the solution $Q_{t}\left(Q_{0}\right)$ nor a criterion of such choice for the function $w$, evidently, the function $w$, (the solution of equation(11)), may be represented by the following equation

$$
V(\zeta)=\int_{\Omega} k(\zeta, \xi) w(\xi) d \zeta
$$

where $\zeta, \xi \in \Omega \subset R^{n}$. Differentiating formula (12), the left-hand and right-hand sides with respect to the (9) we obtain

$$
w(\xi)=\int_{\Omega} k(\zeta, \xi) w(\xi) d \zeta
$$

Here $\tilde{k}=\frac{\partial \omega}{\partial \xi} F(\xi)$, and $\xi \equiv \Theta$. The conclusion from this is that, the equation (11) will be resolved if its right side is a solution of the Fredholm's integral equation (13). For detailed proof see e.g. [15]. Here kernel $\omega(\zeta, \xi)$ is given by a sum of series, i.e.

$$
k(\zeta, \xi)=\sum \lambda_{i} \varphi_{i}(\zeta) \overline{\varphi_{i}(\xi)}
$$

where $\left\{\varphi_{k}(\xi)\right\}$ is a complete orthonormal set in $L^{2}(\bar{\Omega})$ and $\left\{\lambda_{k}\right\}$ is a set of numbers $\lambda_{k}=\mu_{k}+\bar{\mu}_{k}$ and $\mu_{k}$ is eigenvalue for the kernel $\tilde{\omega}$. A common way of regarding this fact is to suppose that the domain $\bar{\Omega}$ is the attractor for the singular point $\Theta=0$ of the equation (11).

In view of the solution for the perturbation equation (9) it is clear that in some neighborhood $\Omega$ of the singular point $\Theta=0$ condition for all $\lambda_{k}<0$ holds. In this case the neighborhood $\Omega$ is an attraction region. From equation (13) we deduce some results about boundary of the domain $\Omega$.

Obviously, the boundary is a closed surface $S=\bar{\Omega}-\Omega$ such that if $\xi \in S$ then $g(\xi)=0, \quad$ i.e., $\frac{\partial v(\xi)}{\partial \xi} F(\xi)=0, \quad \xi \in S$. Also assume that on the surface the following conditions are satisfied: $(i)$ it is surface in phase space such that the particle beam can not go through it i.e., it plays the roll of the impenetrable wall; (ii) it contains whole trajectory of dynamical system (11).

To be more clear we consider the following example.

EXAMPLE: Let $\left(x_{2}=x_{3}=0, \quad v_{2}=v_{3}=0\right)$ be an integral manifold. Denote $x_{1}=x, \quad v_{1}=v$ and $H=\left(H_{1} 0,0\right)$, thus

$$
\dot{x}=v
$$




$$
\begin{gathered}
\dot{v}=q E \\
\Delta U=-4 \pi q \int_{-1}^{1} f(t, x, v) d v
\end{gathered}
$$

This reasoning yields a simple equation:

$$
\partial_{t} f+v \partial_{x} f-4 \pi q \int_{-1}^{1} f(t, x, v) d v \cdot \partial_{v} f=0
$$

Now taking the solution $f$ in the form $f(t, x, v)=f_{0}(x, v) e^{i \omega t}$ we get

$$
v \partial_{x} f-4 \pi q \int_{-1}^{1} f(t, x, v) d v \cdot \partial_{v} f_{0}=i \omega f_{0}
$$

Remark 1 Taking $U=U_{0}(x) e^{i \omega t}$, we see that $U_{0}=-4 \pi q \int_{-1}^{1} f_{0}(x, v) d v$.

The equation (14) is quasi-linear equation, therefore it will be reduced to a linear equation

$$
v \partial_{x} \Phi-4 \pi q \int_{-1}^{1} f_{0} d v \cdot \partial_{v} \Phi-i \omega f_{0} \partial_{f_{0}} \Phi=0
$$

where $\Phi=\Phi\left(x, v, f_{0}\right)$ is an integral of the linear equation (15) As known, the equation (15) yields

$$
\frac{d x}{v}=-\frac{d v}{8 \pi f_{0}}=-\frac{d f_{0}}{i \omega f_{0}}
$$

Remark 2 The function $f_{0}$ play roll of an independent variable in the equation (5). Now it is easy to establish the existence of an integral basis [20] for equation (15):

$$
\left(8 \pi f_{0} z^{2}+v^{2}, v x-\frac{i \omega}{2} f_{0}^{2}\right)
$$

Remark 3 Without loss of generality it can be assumed that $v \in[-1,1]$, as was assumed in the above.

The solution of Cauchy problem (initial-value problem) is called the solution of equation (14), $f_{0}(x, v)$ satisfying some initial conditions: $f_{0}^{0}=\eta(v)$ for $x=x_{0}$. Here the function $\eta$ is a distribution of the velocity $v$ for some fixed point $x_{0}$. 
Example 4 Let $x=x_{0}=0$ and $\eta(v)=\exp \left(-\frac{v^{2}}{\varepsilon}\right)$ where $\varepsilon$ is some constant. Using the technique of Cauchy problem solvability, we will easily see that the solution $f_{0}$ on the integral manifold have the following form

$$
f_{0}=\frac{i \omega x}{v}-\left\{\frac{m}{2 \pi \Theta}\right\}^{\frac{3}{2}} \exp \left\{-\frac{m}{\Theta}\left[8 \pi L f_{0} x-\frac{1}{2} v^{2}\right]\right\}
$$

where $0 \leq x \leq L, \quad v \in[-1,1]$.

Corollary 5 We have arrived at the following result: The function $f_{0}(x, v)$ possesses the property and the form which it succeeded from the function $\varphi$.Consequently, there exist situations such that the solution $f_{0} e^{i \omega t}$ have the solitonlike form. For this we choose the function $\varphi\left(v, x_{0}\right)$ in accordance with this conditions. E.g., see [17], [18]

Example 6

$$
\varphi\left(x_{0}, v\right)=4 \tan ^{-1}\left\{\exp \left[\left(x_{0}-v t\right) /\left(1-v^{2}\right)^{1 / 2}\right]\right\}
$$

The "classical" problem of the soliton solution can be posed as follows: identify the Lorentz force $F_{l}(E, H)$, such that the motion on the integral manifold will be an asymptotically stable. We are going to consider the problem of the stability in the above mentioned solutions. Assume conditions $x_{1}\left(t_{0}\right)=x_{10}, x_{2}\left(t_{0}\right)=x_{20}, x_{3}\left(t_{0}\right)=$ $x_{30}$; and $v_{1}\left(t_{0}\right)=v_{10}, v_{2}\left(t_{0}\right)=v_{20}, v_{3}\left(t_{0}\right)=v_{30}$ are satisfied. Next we suppose that $\left(0, x_{20}, x_{30}\right) \in \Omega_{x},\left(0, v_{20}, v_{30}\right) \in \Omega_{v}$, where $\Omega_{x}=\left\{x_{i 0}: \sum_{2}^{3} x_{i 0}^{2} \leq 1\right\}, \quad \Omega_{v}=$ $\left\{v_{i 0}: \sum_{2}^{3} v_{i 0}^{2} \leq 1\right\}$ without loss of generality it can be assumed. Thus the perturbed has place in the planes $\left(\left\{x_{2}, x_{3}\right\} \times\left\{v_{2}, v_{3}\right\}\right)$. Let $D=\Omega_{x} \cdot \Omega_{v}$ be a topological multiplying the domains $\Omega_{x}$ and $\Omega_{v}$.

Let $\left\{\varphi_{k}\left(x_{2}, x_{3}\right)\right\}$ and $\left\{\varphi_{k}\left(v_{2}, v_{3}\right)\right\}$ be complete orthonormal sets in $\Omega_{x}$ and $\Omega_{v}$ respectively, then $\left\{\varphi_{k} \Psi_{r}\right\}$ be a complete orthonormal set in D.

Now we can construct the kernel

$$
k=\sum_{i} \sum_{j} \lambda_{i j} \varphi_{i}(x) \Psi_{j}(v) \overline{\varphi_{i}(y) \Psi_{j}(u)},
$$

where $x, y, \in \Omega_{x}, v, u \in \Omega_{v}$. Now by using (12) and (13) we can find the solution of the Cauchy problem (11).

Note that, present techniques are just arriving at the point where this approach can be proved if the phase space of the physical system is limited and is closed, i.e. the phase space is a compact with bound.

\section{TWO-DIMENSIONAL PROBLEM}

In view of the above and numerical algorithms of the solutions, it is clear that simplification of the physical model is necessary to yield practical solutions. 
The perturbation motion is described in the plane which is orthogonal to the main motion. Thus our approach can be improved by simplicity of the two-dimensional problem. In this section we further develop the technique which was proposed above. We shall use z-complex plane to express the disturbance. As before we consider the motion of a beam in a plane $\left\{x_{2}, x_{3}\right\}$ with a velocity $\left\{v_{2}, v_{3}\right\}$. Now we introduce complex variable $z: \quad z=x_{2}+i x_{3}$ and $\dot{z}=\dot{x}_{2}+i x_{3}=V$, then we have the following system

$$
\begin{gathered}
\dot{z}=V \\
\dot{V}=q \bar{E}+\frac{q}{c}[\overline{V H}]=\bar{F}_{l}
\end{gathered}
$$

wherc $\bar{E}=E_{2}+i E_{3}$, and $[\overline{V H}]=[V H]_{x_{2}}+i[V H]_{x_{3}}$.

From these definitions, we immediately obtain the following relation

$$
\frac{\partial f}{\partial t}+V \partial_{z} f+\overline{\bar{i}}_{l} \partial_{V} f=0
$$

By the use of the WKB approximation, that is, the wave packet form, we have

$$
f(t, z, V)=f_{0}(z, V) e^{-i \omega t} .
$$

Substituting (18) in (17), we get

$$
V \partial_{z} f_{0}+\bar{F}_{l} \partial_{V} f_{0}=i \omega f_{0}
$$

Now have to resolve this equation. If we consider the variable $z \in C=$ $\{z:|z|<1\}$, then

$$
f_{0}=\sum c_{k} \varphi_{k}(z)
$$

the set $\left\{\varphi_{i}\right\}$ is some complete orthonormal system in the circle $C$. Let $\left\{\varphi_{k}(z)=r e^{i k \theta}, r=\left(x_{2}^{2}+x_{3}^{2}\right)^{1 / 2}\right\}$ be one. For the orthonormal system we obtain

$$
c_{k}=\int_{C} \varphi_{k}(z) f_{0}(z, V) d z
$$

thus $c_{k}=c_{k}(V)$.

By substituting the value series (20) in (12)we obtain

$$
f_{0}(z, V)=i \omega \int_{C} k(z, \bar{\xi}) f_{0}^{*}(\xi, V) d \xi
$$

$z, \xi \in C, \omega(z, \xi)=\sum \varphi_{i}(z) \varphi_{i}^{*}(\xi)$.

Using the values of the coefficients $\left\{c_{k}\right\}$ we get 


$$
k(z, \bar{\xi})=\frac{1}{\pi} \sum_{n=0}^{\infty}(n+1)(z \bar{\xi})=\frac{1}{\pi} \frac{1}{(1-z \bar{\xi})^{2}} .
$$

Substituting (22) in (21) we get

$$
f_{0}(z, V)=\frac{1}{\pi} \int_{C} \frac{1}{(1-z \bar{\xi})^{2}} f_{0}^{*}(\xi, V) d \xi .
$$

It may be noted that there exists an infinite number of different orthonormal systems the circle $C$, but the functions $k$ for all systems coincide.

It is easy to see that the systern (21) can be written as

$$
\dot{z}=V, \quad \ddot{z}=F_{l} .
$$

Using this equation we immediately obtain the important equation

$$
i \omega f_{0}(z, V)=\int_{C} \tilde{\kappa}(z, \bar{\xi}) f_{0}^{*}(\xi, V) d \xi
$$

where

$$
\approx \widetilde{k}=\frac{d^{2} k}{d t^{2}}\left(\frac{1}{\pi} \frac{1}{(1-z \xi)^{2}}\right) .
$$

We recall, the function $\widetilde{\kappa}$ is the second time derivative of $k$ with respect to the given system (24), i.e.

$$
\tilde{\kappa}(z, \bar{\xi})=\frac{6}{\pi} \frac{\xi^{2}}{(1-z \xi)^{4}} V^{2}+\frac{2}{\pi} \frac{\xi}{(1-z \xi)^{3}} F_{l} .
$$

Then here exist function $f_{0}(z, V)$ such that the integral equation (25) hold. The equation (25) is called the Fredholm integral equation with kernel $\widetilde{\tilde{k}}(z, \xi)$. Here the set $\{i \omega\}$ is the spectrum $\sigma$ of kernel $\tilde{\tilde{k}}$. Examining the differential equations

$$
\begin{gathered}
\frac{d f_{0}}{d t}=\dot{f}_{0} \\
\frac{d \dot{f}_{0}}{d t}=-\omega^{2} f_{0} .
\end{gathered}
$$

We recognize that its characteristic numbers are $\pm i \omega$.Consequently the general solution

$$
f(t, z, V)=c_{1} \sum c_{k}(V) r^{k} e^{i(k \Theta-\omega t)}+c_{2} \sum c_{k}(V) r^{k} e^{i(k \Theta+\omega t)}
$$

where $c_{1}$ and $c_{2}$ are some constants which depend on initial condition. Therefore if it is chosen $c_{2}=0$ and $\omega=i \lambda$, where $\lambda>0$ then the perturbed function 
$f(t, z, v) \rightarrow 0$ as $t \rightarrow \infty$. Thus the bunched beam survive the full energy so that for this condition we have self-focusing and accelerating system.

It can be shown in the usual way, that if $i \omega \in \sigma(\widetilde{\widetilde{k}})$ be such that

$$
\operatorname{Im} \sigma(\widetilde{\tilde{k}}) \succeq \alpha>0
$$

then the integral manifold $\left\{x_{1}, x_{2}=0, x_{3}=0\right\}$ is self-focused and acceleration.

Indeed, under condition (27) we can construct the function $\varsigma=f_{0} \vec{f}_{0} \geq 0$ such that its time derivative with respect to the given system (21), (i.e. the expression $\frac{d \varsigma}{d t}=$ $\left.-2|\omega| f_{0} \bar{f}_{0}\right)$, is positive everywhere except at the singular point where it vanishes. Under this condition, consequently $\varsigma(z, V) \rightarrow 0$ as $t \rightarrow \infty$.

Furthermore, the boundary of asymptotically stable is defined by a condition

$$
\int_{C} \approx \tilde{k}_{(z,}(z) f_{0} d \xi=0
$$

Thus the equation (28) is a common way of looking for the electro-magnetic field resolving the $2-\mathrm{D}$ problem 1 .

\section{THE STABILITY OF THE SOLUTION OF VLASOV-POISSON EQUATIONS IN THE SENSE OF TWO METRIC}

As well known, the stability of local structures or solitons by two metric have important physical sense. Thus the solution of the Vlasov-Maxwell system is the stability for the metric $\rho_{0}$ and $\rho$ if for all $\varepsilon>0$, there exists $\delta(\varepsilon)>0$ such that from $\rho_{0}\left(x_{0}, v_{0}\right)<\delta$ follows $\rho(f(t, x, v))<\varepsilon$ for all $t>0$. If in addition $\rho(f(t, x, v)) \rightarrow 0$ as $t \rightarrow \infty$ then we have the asymptotically stable. Let $\rho_{t}=\int_{D_{0}}|f(t, x, v)|^{2} d \mu=\|f\|_{l_{2}}$ be the norm of the function in a space $L^{2}(D)$, then $\rho_{0}=\int_{D_{t}}|f(0, x, v)|^{2} d \mu$, where $\mu$ is some measure in $L_{2}(D)$. From the above mentioned result about $\rho_{t}$ we deducc

$$
\rho_{t}=\int_{D_{t}}|f(t, x, v)|^{2} d \mu=\int_{D_{t}}|f(0, x, v)|^{2} \exp \int_{0}^{t} \operatorname{divX} d \tau d \mu
$$

here $\operatorname{div} X$ is a divergent of the vector field $X=\left\{v, F_{l}+G(t, x, v)\right\}$ and $G(t, x, v)$ is some disturbance of the Lorentz force (e.g., it may be collision of the particles). Notice there exists some constant $B$ such that an inequality

$$
\exp \int_{0}^{t} \operatorname{div} X d t \leq B e^{-\alpha t}
$$


iff $\operatorname{Re} \sigma(k) \leq-\alpha<0$. The inequality (29) yields: $\rho_{t} \leq B \rho_{0} e^{-\alpha t}$ and $\rho_{t} \rightarrow 0$, as $t \rightarrow \infty$; as only $\operatorname{Re} \sigma(k) \leq-\alpha$ if $\rho_{0}<\delta$.

It is easily seen, that norm $\rho$ in the Hilbert space $L^{2}(D)$ have to deal with volume and is determined up to measure zero. This volume may be $m$-dimensional chaotic attractor in some dissipative phase space.

\section{REFERENCES}

1. A.A. Vlasov, Many-Particle Theory and its Application to Plasma Physics, (1961).

2. E.g. see $[3]-[14]$ and references therein. Also see presentations in this workshop.

3. C.M. Ankenbrandt etal., Status of muon collider research and development and future plans, Phys. Rev. ST Accel. Beams 2, 081001 (1999), and references therein.

4. A.M. Skrinsky and V.V. Parkhomchuk, particles, Sov.J.Part.Nucl.,12,p.223(1981).

5. Z. Parsa, Muon Storage Rings - Neutrino Factories, in Procds. of the Workshop on the Next generation Nucleon decay and Neutrino detector AIP-Press (2000).

6. Z. Parsa, ed., Future High Energy Colliders, AIP CP 397, AIP-Press, NY (1997).

7. Z. Parsa, Ionization cooling and Muon Dynamics, AIP CP 441, 289-294 (1997).

8. Z. Parsa, New High Intensity Muon sources and Flavor Changing Neutral Currents, World scientific Publishing, pp 147-153 (1998).

9. Z. Parsa, ed., Beam Stability and Nonlinear Dynamics, AIP CP 405 (1997).

10. Z. Parsa, Lasers and Future High Energy Colliders, STS-Press, pp 823-830 (1997).

11. B. Kamal, W. Marciano, Z. Parsa, Resonant Higgs enhancement at the first muon collider, AIP CP441, pp174- (1997); ibid, AIP 435 pp567-662 (1997).

12. Z. Parsa, $\mu^{+} \mu^{-}$Collider and Physics Possibilities (1993) (unpublished).

13. Z. Parsa, Polarization and Luminosity requirements for the First Muon Collider, in 8th Advanced Accelerator Concepts AIP CP 472, pp. 251 - 259 (1999).

14. Z. Parsa, Muon Dynamics and Ionization Cooling at Muon Colliders, Procd. of EPAC98, Stockholm, Sweden, Vol 2, pp.1055-.

15. V. Zadorozhny, On the arise of the deterministic chaos in dynamical systems. Proceedings of ICNPAA-98, v.2, p.797-803 (1999).

16. A.V. Balakrishnan, Introduction to optimization theory in a Hilbert space, SpringerVerlag (1971).

17. Z. Parsa, Topological solitons in physics, Am.J.Phys. Vol.47. N.1, p.56-62 (1979).

18. Z. Parsa, Nonlinear Dynamics - Maps, Integrators and Solitons, Procds. of 2nd Int'l Conf. on Nonlinear Problems in Aviation and Aerospace, Vol. 2, pp. 589-600 (1999).

19. V.F. Zadorozhny, On the stability volume of measure zero in to the dissipative $d y$ namical systems, J. Nonlinear studies,vol.5, n.1, p.115-122, (1998).

20. E. Kamke, Partielle differential gleichungen erster ordnung, Leipzig, (1959). 